\title{
Diferencias intergeneracionales en el Sentido de Comunidad entre un grupo de niños y adultos mayores de la parcela de Cujacal en la ciudad de San Juan de Pasto - Colombia
}

Intergenerational differences in the sense of community of a group of children and older adults of the Cujacal plot in San Juan de Pasto city - Colombia.

Diferenças intergeracionais no sentido de comunidade entre um grupo de crianças e adultos da terceira idade de Cujacal, na cidade de San Juan de Pasto - Colômbia.

DOI: https://doi.org/10.21803/pensam.v12i22.253

Jonnathan Harvey Narváez

https://orcid.org/0000-0002-3023-5156.

Edith Lourdes Hernández

https://orcid.org/0000-0001-9695-4252

\section{¿Cómo citar este artículo?}

Narváez, J. \& Hernández, E. (2019). Diferencias intergeneracionales en el Sentido de Comunidad entre un grupo de niños y adultos mayores de la parcela de Cujacal en la ciudad de San Juan de Pasto - Colombia. Pensamiento Americano, 12(23), 154-168.

DOI: https://doi.org/10.21803/pensam.v12i22.253:

\section{Resumen}

Elsentido de comunidad constituye un fenómenofundamentalal momento de analizar las realidades comunitarias; de allí que su abordaje desde una perspectiva intergeneracional permita el reconocimiento de diferencias y confluencias subjetivas en los participantes, pese a compartir los mismos espacios geográficos. Reconocer las diferencias intergeneracionales entre adultos mayores y niños de la comunidad de Cujacal Centro frente al sentido de comunidad. Se propuso un estudio cualitativo, con enfoque histórico hermenéutico y perspectiva fenomenológica. Se aplican grupos focales y entrevistas a profundidad. Participantes, niños y adultos mayores. Resultados: Se presentan diferenciasy puntos de confluencia. Las diferencias más destacadas se ubican en torno a la conexión emocional compartida, la integración, satisfacción de necesidades y la membresía, mientras que hay confluencias en la percepción de influencia. Se reconocen diferencias intergeneracionales que dan cuenta que el espacio geográfico compartido no determina la misma percepción del sentido de comunidad, de igual manera, se identifica que tanto niños como adultos mayores, no tienen un fortalecido sentido de comunidad, como tampoco comparten escenarios de interacción que les permita su integración.

Palabras Claves: Sentido de Comunidad, intergeneracional, adultos mayores, niños, comunidad.

\begin{abstract}
The sense of community constitutes a fundamental phenomenon when analyzing community realities; hence, its approach from an intergenerational perspective allows the recognition of differences and subjective confluences in the participants, despite sharing the same geographical spaces. Recognize the intergenerational differences between older adults and children in the community of Cujacal Centro compared to the sense of community. A qualitative study was proposed, with a historical hermeneutical approach and phenomenological perspective. Focus groups and in-depth interviews apply. Participants, children and older adults. Results: Differences and points of confluence are presented. The most noticeable differences are around the shared emotional connection, integration, satisfaction of needs and membership, while there are confluences in the perception of influence. Intergenerational differences are recognized that realize that the shared geographical space does not determine the same perception of the sense of community, in the same way, it is identified that both children and older adults do not have a strengthened sense of community, nor do they share interaction scenarios that allow them to integrate.
\end{abstract}

Key words: Sense of Community, intergenerational, older adults, children, community.

* Derivado del proyecto de investigación "Fortalecimiento del sentido de comunidad desde la recuperación de la memoria histórica y los encuentros intergeneracionales en el Sector de Cujacal Centro Corregimiento de Buesaquillo - Pasto" Financiado en la convocatoria de investigación docente, Universidad de Nariño, 2016. 


\section{Resumen}

O sentido de comunidade constitui um fenômeno fundamental no momento de analisar as realidades comunitárias; sua abordagem desde uma perspectiva intergeracional permita o reconhecimento de diferenças e confluências subjetivas nos participantes, apesar de compartilhar os mesmos espaços geográficos. Reconhecer as diferenças intergeracionais entre adultos da terceira-idade e crianças da comunidade de Cuajacal centro em face ao sentido de comunidade. Foi proposto um estudo qualitativo com enfoque histórico-hermenêutico e perspectiva fenomenológica. Foi utilizada a técnica de grupos focais e entrevistas em profundidade. Resultados: Foram notadas diferenças e pontos de confluência. As diferenças mais destacadas se localizam sobre a conexão emocional compartilhada, a integração, a satisfação de necessidades e a filiação, enquanto existem confluências na percepção de influência. São reconhecidas as diferenças intergeracionais que sinalizam que o espaço geográfico compartilhado não determina a mesma percepção do sentido de comunidade, do mesmo modo se identifica que tantas crianças como adultos da terceira-idade não tem o sentido de comunidade fortalecido, como tampouco compartilham cenários de interação que lhes permita sua integração.

Palavras-chave: Sentido de comunidade, intergeracional, adultos da terceira-idade, crianças, comunidade.

\section{Perfil}

Lic. Filosofía Pensamiento Político y Económico, Especialista en Estudios Latinoamericanos, Magister en Investigación Integrativa, Doctor en Ciencias de la Educación, Docente Investigador Universidad de Nariño.

jonnathanharnarvaez@gmail.com

Jonnathan Harvey Narváez Psicólogo

\section{Perfil}

Especialista en Bioética, Magister en Docencia Universitaria, Docente investigadora Universidad de Nariño. 


\section{Introducción}

El concepto de comunidad ha sido definido como un grupo humano enmarcado en un espacio geográfico determinado que comparte, en lo fundamental, comunión de actitudes, sentimientos, tradiciones, usos y patrones lingüísticos comunes correspondientes a una lengua histórica o idioma; con las características propias que le permiten identificarse como tal. Este grupo de personas que vive en un área geográficamente específica comparten actividades e intereses comunes, en las cuales pueden o no cooperar formal e informalmente con el fin de dar solución a los problemas colectivos (Causse, 2009). Por su parte, Sánchez Vidal (1996), plantea que la comunidad es un sistema o grupo social de raíz local, diferenciable en el seno de la sociedad de la cual se es parte con base a características e intereses compartidos por sus miembros y subsistemas que incluyen: localidad geográfica, interdependencia e interacción psicosocial estable, sentido de pertenencia a la comunidad e identificación con sus símbolos e instituciones, todos ellos enmercados en un sentimiento o sentido de comunidad (Meza, 2009).

En los últimos 20 años el sentido de comunidad, como concepto incorporado a la reflexión de los procesos sociales ha incrementado su relevancia a nivel investigativo, debido a que en el contexto actual se refleja una pérdida progresiva de la cohesión, integración, filiación y pertenencia de los individuos a sus comunidades (Guitart \& Sánchez, 2012); razón por la cual, el estudio del sentido comunitario y la búsqueda de estrategias que lleven a su fortalecimiento se ha convertido en una preocupación central en la Psicología Comunitaria y otras disciplinas llamadas a comprender la organización social y los diversos fenómenos que den cuenta tanto de factores protectores como de problemas sociales.
Tal es el caso de la comunidad de Cujacal Centro, ubicada en el corredor oriental del Municipio de Pasto en el Corregimiento de Buesaquillo, zona de amplio impacto por la construcción de la nueva vía panamericana que fracciona en dos sectores su territorio, además por el crecimiento urbano de la ciudad que deja la cabecera veredal ya a $6 \mathrm{mi}-$ nutos del casco urbano. El sector de Cujacal Centro al limitar con los Barrios Sindagua y Nueva Sindagua, éste último proyecto de vivienda social en el programa del Ministerio de Vivienda, en el cual fueron beneficiadas personas de estratos socioeconómicos 0 y 1 ha venido presentando nuevas dinámicas asociadas a la inseguridad, creciente consumo de SPA en población juvenil y cambios en las prácticas de sostenimiento familiar, del sector agricola al de construcción. Adicionalmente, en la comunidad se identifican procesos de conurbación que impactan las dinámicas de organización comunitaria, integración, sistema de creencias y prácticas sociales, por cuanto sus habitantes perciben amenazante en su estructura social el influjo de los imaginarios y prácticas de la ciudad que se yuxtaponen abruptamente desde la población juvenil a las dinámicas históricas de su comunidad, factor que centra el interés del estudio en el análisis de los sentidos de comunidad en adultos mayores y niños, los primeros como receborio de la identidad cultural y comunitaria de la comunidad, los últimos al presenciar un nuevo escenario de interacción comunitaria, lo que inscribe la intencionalidad del artículo, en proponer un análisis del sentido de comunidad en el marco de los estudios intergeneracionales.

Dilthey, (1875, citado por Paolo, 1999) define la generación como una entidad constituida por un grupo de individuos que han compartido una experiencia histórica, vivencia de la cual se deriva su orientación moral y un destino común; mientras Ojeda y López (2017), 
señalan dentro de las relaciones intergeneracionales a aquellas mediadas por algún componente cultural como los valores, las prácticas y las acciones, que favorecen la interacción de personas pertenecientes a distintas generaciones, tal es el caso de los niños y los adultos mayores. Razón por la cual se asume el concepto de generación en un sentido histórico, es decir, desde la perspectiva del grupo de edad, como una cohorte de individuos que comparten similar identidad etaria y que se constituyen como grupo social (Paolo, 1999), de modo, que las generaciones se entienden como un conjunto de individuos que comparten un nicho de experiencias que les sujetan a hechos históricos compartidos, y que les circunscriben a una fase del ciclo vital (Caballero \& Baigorria, 2013).

En cuanto al sentido de comunidad, Sarason (1974; citado por Montero, 2004), fue el primero en usar dich noción, refiriéndose a ésta como la percepción de similitud con otros, una interdependencia consciente, una voluntad de mantener esa relación de influencia dando o haciendo a otros lo que se espera de ellos; por ende, se convierte en aquel sentimiento de que se es parte de una estructura mayor, estable y de la que se depende (Montero, 2004). Asimismo, el sentido de comunidad involucra a las personas en acciones para la resolución de problemas, generando en el actuar colectivo posibles soluciones efectivas a los diversos problemas de la comunidad (Távara, 2012). En esa perspectiva McMillan y Chavis (1986) proponen cuatro componentes con el fin de explicar el concepto de sentido de comunidad, los cuales son, membresía o pertenencia, influencia social, integración y satisfacción de necesidades y conexión emocional compartida.

El componente de Membresía o Pertenencia consiste en el sentimiento de haber invertido parte de sí mismo en la comunidad, y de per- tenecer a ella. Concretamente, esto conlleva (a) la delimitación de una frontera entre los miembros y los que no lo son, (b) la existencia de un sistema de símbolos compartidos, (c) la experiencia de seguridad emocional, (d) la inversión personal en la comunidad y, finalmente, (e) el sentimiento de pertenencia e identificación (McMillan \& Chavis, 1986); es decir, este componente abarca la historia y la identidad social compartida por los miembros, los símbolos comunes, la seguridad y el apoyo emocional, la inversión personal en la comunidad; además de los derechos y deberes provenientes de ser parte de esta (Montero, 2004).

El componente de Influencia se refiere al poder que los miembros ejercen sobre el colectivo y recíprocamente el poder de las dinámicas del grupo sobre sus miembros (McMillan \& Chavis, 1986). Este componente implica la cohesión y la unidad del grupo, esta cohesión depende de si el grupo tiene o no influencia sobre sus miembros (Meza, 2009) y hace referencia a la capacidad, tal como es percibida, de inducir a otros a actuar de una cierta forma, así como de ser consultados o de que su opinión sea escuchada y tenga un alto valor en la comunidad (Montero, 2004).

El componente de Integración y satisfacción de necesidades tiene que ver, con los valores compartidos por los miembros del grupo;y se refiere al intercambio de recursos para satisfacer las necesidades de los integrantes (McMillan \& Chavis, 1986); es decir, tiene que ver con los beneficios que la persona recibe por el hecho de pertenecer a la comunidad. Estos beneficios pueden ser en términos de status, respeto, valores compartidos, popularidad y ayuda material y psicológica en momentos de necesidad (Maya, 2004). Asimismo, el componente de Conexión Emocional, en el cual los miembros reconocen la existencia de un lazo compartido, y destacan éste vínculo 
como el resultado del contacto positivo prolongado y de participar de experiencias y una historia común (Maya, 2004). Según McMillan y Chavis (1986), este es el componente fundamental en el Sentido de Comunidad.

Finalmente, los estudios intergeneracionales posibilitan el conocimiento de las interacciones sociales en las dimensiones macro y microsocial (Ojeda \& López, 2017), de allí el interés del presente artículo de describir las diferencias intergeneracionales como campo de oportunidad en el reconocimiento de los roles sociales de los grupos etarios frente al sentido de comunidad, y proponer como marco de reflexión la existencia de diferencias sustantivas en los componentes de pertenencia, conexión emocional, influencia e integración de acuerdo a las vivencias generacionales como individuales desde las cuales se construyen campos de representación simbólica y valoración del entorno.

\section{METODOLOGÍA}

\section{Paradigma}

El paradigma en el que se fundamentó este proyecto de investigación es de tipo cualitativo, por cuanto es una herramienta que intenta hacer una aproximación global de las situaciones sociales para explorarlas, describirlas y comprenderlas, teniendo en cuenta los conocimientos que tienen las diferentes personas involucradas en ella, lo cual supone que los individuos interactúan, con el fin de obtener un concepto común de la realidad que comparten (Bonilla y Rodríguez, citado en Del Canto, 2012).

De acuerdo a Martínez (2006), la investigación cualitativa trata de identificar la naturaleza profunda de las realidades, su estructura dinámica y relaciones que se establecen, es decir da razón plena del comportamiento y
Diferencias intergeneracionales en el Sentido de Comunidad entre un grupo de niños y adultos mayores de la parcela de Cujacal en la ciudad de San Juan de Pasto - Colombia

manifestaciones, con el fin de recoger datos, categorizarlos e interpretarlos; por lo tanto, considera que el conocimiento es el resultado de una relación compresiva entre el investigador y la realidad abordada. En efecto, la investigación tuvo como fin reconocer las diferencias intergeneracionales en la percepción del sentido de comunidad desde un marco de indagación discursivo, que permita el reconocimiento subjetivo de cada participante frente a los componentes del sentido de comunidad como construcción social, por tanto, se enfatizó en explorar de manera sistemática los conocimientos, actitudes y valores que comparten los individuos en un determinado contexto social (Bryman, 1988, citado en Bonilla y Rodríguez, 1997).

En coherencia, el trabajo realizado siguió el enfoque histórico-hermenéutico, el cual según López (2001), busca comprender e interpretar un fenómeno o realidad en un contexto concreto, que para fines de la investigación radica exclusivamente en el reconocimiento de las diferencias intergeneracionales en el sentido de comunidad del entorno veredal de Cujacal Centro.

\section{Método Fenomenológico}

De acuerdo a Martínez (1998) la fenomenología centra su interés en el significado colectivo que los participantes le otorgan a su experiencia, devalando las estructuras sociales que constituyen prácticas comunitarias y establecen lógicas de significación, postulado coherente con lo planteado por Marí, Bo, y Climent, (2010) para quien "las estructuras fenomenológicas no son directamente observables y es necesaria la construcción de modelos de evaluación que reflejen las intenciones y significados de los actores" (p.115) de manera que la función social de investigador es facilitar el acceso a lo vivido en la compilación de la experiencia tal como se va produciendo socialmente. 


\section{Unidad de Análisis}

Adultos mayores y niños pertenecientes a la comunidad de Cujacal Centro - Municipio de Pasto, Colombia, cuyo nacimiento, residencia actual y vivencia histórica se reporte dentro del entorno veredal. Se entiende por vivencia histórica, cuando una persona ha vivido más del $80 \%$ del ciclo vital o de la edad cronológica dentro de una comunidad.

\section{Sujetos de Estudio}

32 niños entre los 7 a 12 años y 18 adultos mayores entre los 69 a 78 años, de género masculino y femenino pertenecientes al entorno rural de la comunidad de Cujacal Centro - Municipio de Pasto, Colombia, cuyo nacimiento, residencia actual y vivencia histórica se reporta dentro del entorno veredal.

\section{Técnicas de Recolección de Información}

Los instrumentos que se implementaron en desarrollo de esta investigación corresponden, en primer término a 6 sesiones de grupo focal, herramienta que permite investigar los relatos de las acciones, de modo que se identifiquen y develen experiencias cotidianas organizadas según la racionalidad de la acción; por cuanto indaga la dimensión práctica de los mundos sociales, que se sostienen en los universos discursivos, ésta técnica tuvo como propósito registrar cómo los participantes perciben los componentes del sentido de comunidad teniendo como referencia su vivencia histórica (Aigneren, 2006).

A su vez, se implementaron 18 entrevistas a profundidad a algunos informantes claves, con quienes se realizaron encuentros reiterados cara a cara entre el investigador y los informantes, dirigidos hacia la comprensión de las perspectivas que tienen los informantes respecto de sus vidas, experiencias o situa- ciones, tal como las expresan con sus propias palabras (Robles, 2011); por informante clave entendimos a adultos mayores de amplia tradición en la comunidad y niños que desempeñaban roles comunitarios, deportivos o de liderazgo en la comunidad. Esta técnica permitió obtener la información necesaria proveniente del discurso de los adultos mayores y los niños quienes relataron sus vivencias comunitarias, sentidos y significados atribuidos a la membresia, la integración, conexión emocional e influencia comunitarias.

\section{Procedimiento}

Inicialmente, se realizó el primer contacto con los participantes a quienes se expuso la naturaleza y relevancia de la investigación, sus responsables e implicaciones, de modo que se garanticen los elementos éticos para que los interesados tomen sin coacción alguna, la decisión de participar del presente estudio dando lugar a la firma del consentimiento informado para los adultos y el asentimiento informado para los menores de edad, donde se incluyen condiciones de participación, las cuales fueron respetadas a lo largo del trabajo de campo e informe de investigación. Desde ésta perspectiva, dado el trabajo con menores de edad, se acordó con los padres de familia los terminos de participación y los diversos escenarios de levantamiento de información que tendrían lugar en el estudio.

Una vez establecidos los acuerdos y contando con las respectivas autorizaciones y consentimientos, se adelantó una inserción en el contexto de interacción comunitaria de los sujetos de estudio, generando escenarios de familiarización mediados por la observación independiente, prosiguiendo con la implementación del trabajo de campo en la aplicación de las técnicas de grupo focal y entrevista a profundidad. Una vez, terminado el trabajo de campo se implementó el plan de análisis 
de información consistente en la reducción, organización de información y emisión de conclusiones desde la elaboración de unidades de significado general que permitieron la contestación de las preguntas orientadoras del estudio así como de los objetivos.

\section{Plan de Análisis de Información}

Por análisis de datos cualitativos se entiende el proceso mediante el cual se organiza y sistematiza la información recogida por los investigadores para establecer relaciones, interpretar, extraer significados, comprensiones y derivar conclusiones (Spradley, 1980). El análisis de información de los datos cualitativos de la presente investigación siguió tres etapas, como sugieren Rodríguez, Quiles \& Herrera (2005).

\section{a) Reducción de Datos}

Comprendió la separación de unidades de contenido en las dimensiones temática, conversacional y social de acuerdo a los ejes de análisis propuestos en el estudio; dichos ejes favorecieron la identificación y clasificación de elementos y facilitaron el proceso de categorización, codificación y simplificación de la información según el interés del estudio y objetivos del mismo (Spradley, 1980).

\section{b) Elaboración de Unidades de significado general}

Para Marí et al. (2010) se trata de recoger las diversas unidades de contenido agrupándolas en porciones que formen una unidad de significado bien desde un eje de análisis previsto anticipadamente o categorías emergentes del trabajo de campo. Una vez obtenidas las unidades de significado relevantes para la investigación se buscó criterios teóricos que permitan agrupar algunas de estas unidades de significado a través de una matriz de organización categorial, cuya disposición refleja la
Diferencias intergeneracionales en el Sentido de Comunidad entre un grupo de niños y adultos mayores de la parcela de Cujacal en la

ciudad de San Juan de Pasto - Colombia

relación fenomenológica de los hallazgos.

Posterior a la identificación de las unidades de significado general y las relaciones fenomenológicas entre las categorías y microcategorías de análisis, se establecieron relaciones teóricas a fin de develar el horizonte interpretativo de la vivencia procedente de narrativas, acciones e interacciones de adultos mayores y niños frente al fenómeno del sentido de comunidad (Rodríguez, Quiles \& Herrera, 2005). En el proceso de clasificación se estableció un marco de relación fenomenológica de las categorías identificadas, desde marcos interpretativos que reconocen el valor de la historicidad y la experiencia subjetiva de los grupos intergeneracionales participantes.

c) Generación de resultados y conclusiones.

Constituye la fase de consolidación teórica, discusión crítica con otros postulados y el establecimiento de conclusiones generales (Rodríguez, Quiles \& Herrera, 2005). Se partió de un análisis fenomenológico que da cuenta de la vivencia subjetiva de los participantes y su campo de representación, articulando en el análisis los postulados teóricos del sentido de comunidad, así como emergentes interpretativos resultantes del análisis de la vivencias intergeneracionales.

\section{Consideraciones éticas}

El proyecto del que se deriva el estudio fue sometido al Comité de Ética de la Universidad de Nariño el cuál dió aval al mismo, dictaminando la viabilidad del consemiento y asentimiento informado de la investigación, el cual garantizó los derechos de confidencialidad, libertad de participación y acción sin daño.

\section{RESULTADOS}

Para la comprensión del sentido de comu- 
nidad y el establecimiento de las diferencias intergeneracionales desde una perspectiva cualitativa, el estudio se orientó desde las cuatro componentes propuestos por McMiIlan y Chavis (1986) y Sarason (1974), mismos que fueron indagados en el sujeto de estudio desde un marco de indagación discursivo.

En relación a la membresía, se atienden los criterios propuestos por (Maya, 2004; McMiIlan y Chavis, 1986), desde los cuales se identifican importantes elementos diferenciales entre los grupos poblacionales del estudio. En los niños, se presenta una concepción de parcela amplia, es decir, para ellos la comunidad es el conjunto de personas con quienes interactúan, sin darle relevancia a los límites geográficos "yo soy de acá y tengo amigos de muchas partes", mientras que los adultos mayores tienen conocimiento de cuales son los límites geográficos que tiene su comunidad, además identifican las familias con quienes se construyó el nicho simbólico de la comunidad "Cujacal tiene historia, los Martínez, Delgado y Merchancano fundaron el pueblo que va desde el puente de tabla hasta la cuesta alta". En cuanto a la percepción de seguridad emocional, los adultos mayores no se sienten involucrados en la dinámica comunitaria, encontrándose las necesidades afectivas insatisfechas "ya uno de viejo no importa en la comunidad, poco lo tienen en cuenta"; por su parte los niños, conciben que la comunidad les ofrece espacios donde pueden expresar sus sentimientos, ser reconocidos y protegidos, también valoran espacios recreativos, culturales y deportivos donde son satisfechas sus necesidades afectivas "yo me siento contento de mi sector porque tenemos todo y mis papás me sacan al parque a jugar y vienen las escuelas de arte".

En relación con el sentido de pertenencia e identificación, los adultos mayores conservan un sistema de creencias asociado a la histo- ria de la comunidad, y tienen expectativas de filiación colectiva con sus pares generacionales, desde donde integran un punto de referencia en su identificación y pertenencia a la comunidad "en la comunidad siempre hemos sido colaboradores, varios apoyamos la construcción del acueducto por eso a veces nos reunimos a recordar"; al contrario los niños, no tienen una expectativa sujeta a una identidad común, entre tanto los procesos de integración son más amplios, reconocen que hacen parte de un entorno veredal, pero su adhesión y pertenencia es más de filiación generacional que de identidad comunitaria "yo conozco a muchos niños porque jugamos en la escuela o en la cancha, pero esos niños vienen de varias partes".

Existe una amplia diferencia en cuanto a la historia y sistema simbólico compartido, los adultos mayores tienen un amplio repertorio de la memoria histórica y cultural de la comunidad, por tanto expresan mayor sensibilidad frente a acontecimientos históricos de la comunidad y reconocen los símbolos religiosos y de paisaje natural propios de la vereda "acá la patrona es María Auxiliadora que la trajimos en el 45 desde lejos" "naturales hay varios lugares importantes en el nacimiento del acueducto o el valle de las piedras"; por su parte lo niños, no comparten un sistema simbólico relacionado con su identificación y reconocimiento de la comunidad, destacan como símbolos culturales sólo la fiesta patronal, desconociendo la construcción histórica, la memoria oral y paisaje natural asociado, dinámica comunitaria que se soporta en el cambio de los procesos comunicacionales entre los roles familiares, y el acceso de los niños a la tecnología y la internet.

Se encuentra una posición común entre los grupos poblacionales, en relación a la inversión personal, donde no se reporta en ninguno de los grupos etarios, esfuerzo por hacer 
parte de la comunidad, siendo claro, que los dos grupos se sienten parte vinculante de su comunidad por el hecho de residir en ella, no por compartir significaciones e imaginarios colectivos comunes. En cuanto al componente de la influencia, los niños consideran que sus acciones no afectan a su comunidad, sin embargo, destacan que las acciones de los adultos sí "los niños todavía no somos grandes, nuestros papás si hacen cosas por el pueblo"; en el mismo sentido, los adultos mayores, reportan que han ido perdiendo con el envejecimiento la influencia en la comunidad, afirmando que tanto su participación como el conocimiento de la comunidad son subutilizados por la población adulta media que ejerce liderazgo e influencia en todos los niveles "ya uno para los jóvenes no vale nada, antes se respetaba a los mayores ahora ya no servimos". En cuanto al ejercicio del poder y la influencia, para los niños es normalizado que dicho rol sea ejercido por los adultos, mientras los adultos mayores expresan niveles de exclusión social en la toma de decisiones, lo que incide en su percepción de cohesión comunitaria. Situación que da cuenta además de la baja autoestima colectiva de los adultos mayores, quienes presentan percepciones negativas frente a su propia valía, desconocen sus aportes a la comunidad y restan valor a su experiencia como aportes comunitarios.

En referencia a la integración y satisfacción de necesidades, los niños se sienten vinculados a un núcleo común liderado por los adultos, el cual logra satisfacer necesidades afiliativas, recreativas, culturales y educativas; mientras los adultos mayores, reportan un nivel más bajo de integración, y expresan que la comunidad logra parcialmente satisfacer sus necesidades recreativas, de afiliación y de salud "ahora ya no hay los espacios de antes, algunas veces nos llevan a celebrar a los adultos mayores pero rara vez". Asimismo, los adultos mayores consideran que la comuni- dad no logra transformar sus necesidades en metas colectivas, por cuanto priorizan en las necesidades de los niños, sobre las del resto de miembros de la comunidad. Adicionalmente, no se encuentran reportes verbales que den cuenta de la capacidad de niños y adultos mayores en la satisfacción de sus necesidades por sí mismos.

En cuanto a la conexión emocional en la que se identifican lazos afectivos más estrechos entre los niños y la comunidad lo que explica una positiva percepción de apoyo social, contraria a la percepción de los adultos mayores, para quienes no existen escenarios que permitan la búsqueda conjunta de decisiones colectivas que repercutan en su integración y bienestar "cuando uno es viejo ya no lo tienen en cuenta para nada, a veces somos estorbos para las familias". Al respecto, se observa que los dos grupos no hacen parte de la búsqueda conjunta de soluciones a los problemas de la comunidad, ni que perciban las mismas necesidades, situación que dificulta el intercambio de recursos y la creación de redes intergeneracionales que soporten relaciones de mutualidad entre adultos mayores y niños. En efecto, la comunidad no se soporta en redes comunitarias ni grupos de interés que permitan la convergencia de las necesidades e intereses de los diversos grupos, pese a las diferencias reportadas en la percepción de apoyo social.

En relación a los procesos socioafectivos, el estudio evidencia la existencia de diferencias sustanciales, los niños sienten mayores niveles de reconocimiento y de apoyo "yo soy feliz en el pueblo porque acá nos quieren arto a los niños", mientras los adultos mayores afirman vivir procesos de exclusión social, percepción que minimiza sus niveles de participación y agudiza la sensación de bajo apoyo social y satisfacción de necesidades. Finalmente, se destaca que las vivencias individuales y ge- 
neracionales presentes determinan las significaciones que tanto adultos mayores como niños dan a su experiencia de comunidad, siendo relevante para las partes el reconocimiento afectivo y el soporte social que pueda brindarles el nicho comunitario.

\section{DISCUSION}

Gracia y Herrero (2006) proponen que la comunidad como sistema geográfico actúa como un elemento de conexión entre la persona y su estructura social, no obstante, el estudio da cuenta de una variación entre los niños y los adultos mayores en diversas dimensiones del sentido de comunidad, pese a compartir el mismo espacio geográfico. En éste nivel, para los primeros la dimensión territorial y geográfica no se constituye en un elemento relevante de membresía, mientras para los segundos dicha dimensión se articula con la memoria oral de la comunidad y favorece su identificación y pertenencia (Gardner \& Davis, 2014); Es posible que los lugares físicos para los niños y adolescentes, ya no representen la importancia que los adultos le dan. Hoy en día en una sociedad digital, virtual, gracias al internet, los jóvenes navegan por sitios o lugares virtuales lo que puede estar relacionado con esa falta de conexión. Los niños se identifican con lugares y personas que ya no hacen parte de su entorno más cercano y real, lo que les lleva a estar más conectados con extraños que con sus propios vecinos, padres o abuelos (Gardner y Davis, 2014).

Frente al sentido de comunidad Camargo y Palacio (2017), sostienen que está relacionado de manera intrapsicológica con la capacidad del individuo de establecer relaciones íntimas dentro de la comunidad, como el tiempo de convivencia, la participación activa en reunionesy la filiación a grupos de interés; al respecto, los niños se perciben más integrados a la comunidad, en la medida que ésta les ofrece varios espacios interactivos, situación opuesta a los adultos mayores quienes se perciben menos integrados al no existir espacios dónde se encuentren vinculados y que les permitan conservan un rol activo dentro de la comunidad.

En relación a la membresía y pertenencia, de acuerdo a Montero (2004) este componente consiste en el sentimiento de haber invertido parte de sí mismo en la comunidad y de pertenecer a ella. Se identificaron diferencias importantes en cuanto a la no existencia de símbolos compartidos y como punto de confluencia que ninguno de los grupos intergeneracionales generaran una inversión personal en la comunidad. En cuanto a los adultos mayores logran delimitar las fronteras entre quienes son reconocidos como miembros de la comunidad y no, mientras que los niños establecen redes comunitarias ampliadas y su concepción de membresía va más allá de los limites territoriales y comunitarios (Maya, 2004); es decir, en referencia a lo postulado por Montero, (2004) los adultos mayores y los niños no tienen una historia e identidad social compartida, lo que implica ausencia de los símbolos comunes, y diferencias en la percepción de seguridad y el apoyo emocional, así como de los derechos y deberes derivados del ser parte de una comunidad (Montero, 2004).

Lo anterior, concuerda con lo propuesto por Cueto, Espinosa, Guillén, Seminario, (2015) quiénes conciben que el sentido de comunidad ofrece un balance subjetivo respecto al sentido de pertenencia, los procesos socioafectivos y las vivencias de los individuos o grupos respecto a la satisfacción de sus necesidades y el reconocimiento. Supuesto evidenciado en los resultados del estudio, donde a modo global existe un mayor sentido de pertenencia de los adultos mayores por su comunidad consecuencia de la vivencia y par- 
ticipación en la construcción histórica de la comunidad de Cujacal, acumulada en su memoria histórica y cultural, la cual se ve menguada por la percepción de exclusión social y socioafectiva; contrario a los niños quienes desconocen marcos de referencia históricosy simbólicos en su comunidad, lo que expresa su poca identificación con la membresía.

En cuanto a la influencia, Montero (2004) sostiene que representa al poder que los miembros ejercen sobre el colectivo y recíprocamente al poder de las dinámicas del grupo sobre sus miembros, factor asociado con la cohesión y la unidad del grupo la cual depende de si el grupo tiene o no influencia sobre sus miembros; en el estudio se evidencia que tanto adultos mayores como niños reconocen que la capacidad de influencia la concentran los adultos medios y perciben que son influenciados permanentemente por las decisiones de ellos; generando malestar para algunos adultos mayores el hecho de perder con el paso de los años su nivel de influencia para verse relegados por las dinámicas impuestas por los adultos medios; en el caso de los niños, asumen de manera receptiva la influencia de los adultos, al verlos como autoridad moral y formativa de la comunidad (Ramos, 2014). En conformidad a lo propuesto por Seminario (2014) teniendo como marco de interpretación una estructura de influencia centro-periferia, los individuos o adultos productivos están ubicados al centro mientras los grupos o individuos que requieren cuidado se ubican en la periferia, postulado que contrasta los reportes verbales de adultos mayores y niños teóricamente ubicados en la periferia, cumpliendo un rol pasivo en las dinámicas de grupo, lo que explica el locus de control bajo.

Lo anterior, corrobora además lo planteado por Ramos y Maya (2014) quienes afirman que la influencia tiende a ser el componente
Diferencias intergeneracionales en el Sentido de Comunidad entre un grupo de niños y adultos mayores de la parcela de Cujacal en la

ciudad de San Juan de Pasto - Colombia

que se presenta más débil en la mayoría de estudios realizados frente al sentido de comunidad, lo que explica la sensación de que las personas no tienen el poder para influir en su entorno y mucho menos en los demás, dejando para el caso del estudio, que tanto niños como adultos mayores crean que son sólo los adultos medios o individuos con capacidad productiva son quienes tienen la capacidad de decidir sobre algunas cosas que suceden en la comunidad; situación que genera una insatisfacción de las necesidades en los adultos mayores, por cuanto aluden que la estructura comunitaria no favorece el intercambio de valores y recursos. Al respecto, Carmona y Granada (2010) señalan que la integración y satisfacción de necesidades es concebida como un requerimiento imprescindible o esencial para alcanzar una condición de vida positiva y cohesión comunitaria, mientras Maya (2004), lo concibe como un elemento que se relaciona con los valores compartidos por los miembros de un grupo y con el intercambio de recursos para la solución de problemas.

Los adultos mayores perciben una perdida progresiva de su participación y su influencia en la toma de decisiones, lo que obstaculiza el establecimiento común de metas colectivas (Cueto et al., 2015), asimismo, se identifica que la comunidad de Cujacal no brinda a sus miembros la oportunidad y la capacidad de satisfacer sus necesidades por sí mismos, lo que obstaculiza el intercambio de recursos internos y externos. Contexto problemático, cuando Carmona y Granada, (2010) subrayan que la satisfacción de necesidades colectivas se constituye como un componente indicador de la existencia de sentido de comunidad, lo que da cuenta que tanto niños $y$ adultos mayores no se perciben integrados ni parte activa de la comunidad desde indicadores de intercambio, interacción común e integración (Carmona \& Granada, 2010). Adi- 
cionalmente, de acuerdo a lo propuesto por Gracia y Herrero (2006) cabe destacar que el deterioro en los procesos de participación del adulto mayor influye negativamente en la percepción de apoyo comunitario, hecho que reduce la calidad de vida, la satisfacción de necesidades y la conexión emocional, lo que deteriora su vida social y crea un sentimiento de exclusión y desconfianza.

En cuanto a la conexión emocional compartida, representa el componente mediante el cual los miembros de una comunidad reconocen la existencia de un lazo compartido, vinculo que de acuerdo a Maya (2004) es el resultado del contacto positivo prolongado de participar de experiencias y una historia común. La conexión emocional compartida es un concepto que surge a partir tanto de los procesos de interacción en la vida cotidiana de los grupos y comunidades que comparten la historia y las experiencias vividas conjuntamente. Los resultados dan cuenta, de que los adultos mayores y niños de la comunidad de Cujacal no reconocen experiencias socioafectivas positivas a nivel intergeneracional, ni tienen afectos comunes hacia los espacios, grupos de interés o referencias históricas lo que minimiza la percepción de sentido de comunidad (Circado, Gonzáles \& Martínez, 2015) lo anterior, puede explicarse debido a que los dos grupos no solo están separados por cuestiones de edad y ciclo vital, sino porque las prácticas de crianza e imaginarios globales les ubica en intereses totalmente distintos; puesto que para que haya conexión emocional además debe haber empatía debe existir una identificación común que les permita la convergencia relacional y afectiva. De igual manera, Meza (2009) define a este elemento como el reconocimiento por parte de los miembros de un grupo o comunidad de la existencia de un lazo compartido que es el resultado del contacto positivo prolongado y de la participación en experiencias e historias comunes, contacto que se ve menguado por los reportes de exclusión social y los procesos culturales, deportivos y recreativos ofertados de manera exclusiva para los niños.

Lo anterior, corrobora la necesidad de generar programas comunitarios que incentiven las redes sociales de carácter intergeneracional y el fomento del apoyo social, permitiendo el incremento de sentimientos de pertenencia e identidad. En cuanto a la conexión emocional también se destaca la dimensión de relaciones positivas en los niños y la percepción de exclusión en los adultos mayores, que puede ser referida a una menor percepción de apoyo social percibido, tal como lo plantea Vivaldi y Barra (2012) para quien sostiene la existencia de una correlación positiva entre la percepción de apoyo social y la percepción de integración que pueda tener un individuo frente a su grupo de referencia. Además de lo planteado por Hombrados y López (2014) respecto a que la conexión entre vecinos y el apoyo social son aspectos importantes del sentido de comunidad, hecho que explica como la baja percepción en la conexión emocional compartida de los adultos mayores lleva a la disminución de su sentimiento de comunidad y aumenta la percepción de exclusión. Asimismo, el autor señala que en los estratos socioeconómicos bajos, la participación comunitaria, la percepción del apoyo social institucional y la participación individual influyen positivamente en el sentido de comunidad, supuesto corroborado en los reportes verbales del adulto mayor, para quienes la exclusión de los procesos de participación comunitaria disminuye su percepción de influencia social creando sentimientos asociados a la exclusión razón por las cuales se aíslan de la vida comunitaria (Gracia \& Herrero, 2006).

En contraste, la vinculación de los niños a diversos colectivos culturales, deportivos o edu- 
cativos les permite experimentar el apoyo social, situación que favorece la ampliación de redes, la pertenencia activa a grupos y desde ellos una mayor satisfacción con su comunidad, así como el acceso a fuentes alternativas de influencia y apoyo lo que supone una mayor integración con su entorno (Gracia \& Herrero, 2006). De igual manera, conforme a lo planteado por McMillan (2011), las personas que adquieren control sobre su entorno tienen mayor identificación y pertenencia a él, en efecto, el estudio evidencia la perdida de control social en los adultos mayores, dados sus menguados procesos de participación en la vida comunitaria, razón por la cual pese a reconocer que tienen una historia común han legado la responsabilidad de la comunidad en los adultos medios, suprimiendo relaciones de influencia bidireccional, pues conciben que los adultos medios los influyen $y$ afectan mientras ellos progresivamente son relegados. Al respecto, las experiencias de participación efectivas, favorecen en niños o adultos mayores tanto la pertenencia como la integración a la comunidad.

A diferencia McMillan y Chavis, (1986) y Sarason (1974) Soto-Johnson, Yestness, y Dalton, (2008) propone que el sentido de comunidad se describe desde cuatro características, coherencia, cohesión, cuidado y contacto, siendo el espacio de interacción el que favorece el contacto como principal dimensión del sentido comunitario; conforme a lo anterior, el estudio evidencia que los niños cuentan con espacios de mayor contacto comunitario que los adultos mayores, lo que favorece la integración de los mismos a la vida comunitaria y mejora de manera significativa la percepción de apoyo social percibido y membresía. A modo global contrario a lo planteado por Kitchen, Williams, y Chowhan (2012) quienes afirman que el sentido de comunidad tiende a ser más alto en los ambientes rurales en comparación con las áreas urbanas, la co- munidad de Cujacal da cuenta de una fragmentación en el sentido de comunidad en la medida que existen distancias intergeneracionales que no permiten la interacción de los adultos mayores y los niños, minimizando los niveles de cohesión y contacto, factores indispensables en sentidos comunitarios fortalecidos.

Finalmente, los resultados del estudio dan cuenta de la necesidad de abordar las dinámicas intergeneracionales en el momento de aproximarse a la comprensión del sentido de comunidad, entre tanto, existen diferencias sustantivas en el campo de representación de los diversos grupos de edad que hacen parte de una comunidad. De igual modo, el sentido de comunidad es dinámico, va mutando en la medida que el individuo inmerso en la vida social, adquiere vivencias y construye significaciones de su entorno que le llevan a valorar su experiencia subjetiva en el marco de un espacio territorial y comunitario, siendo las experiencias individuales y colectivas de acuerdo a la generación histórica a la que se pertenezca, las que determinan los niveles de pertenencia, integración, influencia y conexión emocional de los individuos respecto a sus comunidades. 


\section{Referencias}

Aigneren, M. (2006). La técnica de recolección de información mediante los grupos focales. Universidad de Antioquia. Facultad de ciencias sociales y humanas facultad de ciencias sociales y humanas facultad de ciencias sociales y humanas centro de estudios de opinión. Recuperado de: http:// ccp.ucr.ac.cr/bvp/ texto/14/grupos_focales.htm.

Bonilla, E. \& Rodríguez, P. (1997). Más allá del dilema de los métodos. Bogotá, Colombia: Ediciones UnidadesGrupo editorial Norma.

Caballero, M. \& Baigorri, A. (2013). ¿Es operativo el concepto de generación?. Aposta. Revista de Ciencias Sociales, (56), 1-45.

Camargo, A. \& Palacio, J. (2017). Apoyo social y sentido de comunidad en desplazados y damnificados en el Departamento del Magdalena. Revista Duazary, 14, 35 -44. DOI: http://dx.doi.org/10.21676/2389783X.1735

Carmona, H. \& Granada, H. (2010). Una mirada psicosocial a la solidaridad y a la satisfacción de necesidades. El caso del barrio "La Honda II - Balboa" de Buga. Revista Cooperativismo y desarrollo, 18 (97).

Causse, M. (2009). El concepto de comunidad desde el punto de vista socio -histórico-cultural y lingüístico. Ciencia en su PC, (3), 12-21.

Circado, J., González, L. \& Martínez, D. (2015). Procesos de fortalecimiento, liderazgo y organización comunitaria de la Casa del Espíritu Juvenil Jaime Garzón Localidad La Candelaria - Bogotá (Tesis). Universidad Católica de Colombia, Bogotá. Recuperado de: https://repository.ucatolica.edu.co/ bitstream/10983/6168/1/Procesos\%20de\%20fortalecimiento\%2C\%20liderazgo\%20y\%20organizaci\%C3\%B3n\%20comunitaria.pdf

Cueto, R., Espinosa, A., Guillén, H. \& Seminario, M. (2015). Sentido de Comunidad Como Fuente de Bienestar en Poblaciones Socialmente Vulnerables de Lima, Perú. Psykhe, vol. 25, (1). 1-18. https://dx.doi. org/10.7764/psykhe.25.1.814

Del Canto, E. (2012). Investigación y métodos cualitativos: un abordaje teórico desde un nuevo paradigma.
Ciencias de la educación, 22(1) 181-199.

Gardner, H. \& Davis, K. (2014). La generación APP: Cómo los jóvenes gestionan su identidad, su privacidad y su imaginación en el mundo digital. Barcelona, España: Paidós. 238.

Gracia, E., \& Herrero, J. (2006). La comunidad como fuente de apoyo social: evaluación e implicaciones en los ámbitos individual y comunitario. Revista Latinoamericana de Psicología, 38 (2), 327-342.

Guitart, M. \& Sánchez, A. (2012). Sentido de comunidad en jóvenes indígenas y mestizos de San Cristóbal de las Casas (Chiapas, México). Un estudio empírico. Anales de Psicología, 28(2) 532-540.

Hombrados, I. \& López, T. (2014). Dimensiones del sentido de comunidad que predicen la calidad de vida residencial en barrios con diferentes posiciones socioeconómicas. Psychosocial Intervention, 23 (3). 159-167.

Kitchen, P., Williams, A. \& Chowhan, J. (2012). Sense of belonging and mental health in Hamilton, Ontario: An intra-urban analysis. Social Indicators Research, 108(2), 277-297.

López, H. (2001). un enfoque histórico-hermenéutico y crítico-social en psicología y educación ambiental. Medellín, Colombia: Universidad Pontificia Bolivariana.

Marí, R., M. Bo, R. \& Climent, T. (2010). Propuesta de Análisis Fenomenológico de los Datos Obtenidos en la Entrevista. UT. Revista de Ciències de l'Educació. 113-133.

Martínez, M. (1998). La investigación cualitativa (síntesis conceptual). REVISTA IIPSI, 9, 123-146.

Martínez, M. (2006). La Investigación Cualitativa. Revista de investigación en psicología, 9(1) 123-146.

Maya, I. (2004). Sentido de comunidad y potenciación comunitaria. Apuntes de Psicología, 22(2) 187-211.

McMillan, D. W. \& Chavis, D. M. (1986). Sense of community: A definition and theory. Journal of Community Psychology, 14, 6-23. doi:10.1002/1520-6629(198601)14: 1<6::AID-JCOP2290140103>3.0.CO;2-1 
McMillan, D. W. (2011). Sense of community, theory not a value: a response to Nowell and Boyd. Journal of Community Psychology, 39, 507-519.

Meza, G. (2009). Comunidad y sentido de comunidad (tesis de pregrado). Universidad de Chile. Recuperado de: http://www.tesis.uchile.cl/tesis/uchile/2009/ cs-meza_g/pdfAmont/cs-meza_g.pdf

Montero, M. (2004). Introducción a la psicología comunitaria. Desarrollo, conceptosy procesos. Buenos Aires, Argentina: Editorial Paidós. Recuperado de: http:// saber.ucv.ve/jspui/bitstream/123456789/4207/1/montero-introduccion-a-la-psicologia-comunitaria.pdf

Ojeda, D. \& López, E. (2017). Relaciones intergeneracionales en la construcción social de la percepción del riesgo. Desacatos. Revista de Ciencias Sociales, (54), 106-121.

Paolo Donati, P. (1999). Familias y generaciones. Desacatos. Revista de Ciencias Sociales, (2), 0.

Ramos, I. (2014). La experiencia de múltiples sentidos de comunidad. Psicología Política, vol 48. 47-67.

Ramos, I., Maya, I. (2014). Sentido de comunidad, empoderamiento psicológico y participación ciudadana en trabajadores de organizaciones culturales. Psychosocial Intervention, 23 (3) 169-176.

Robles, B. (2011). La entrevista en profundidad: una técnica útil dentro del campo antropofísico. Revista Cuicuilco, 18 (52), 39-49.

Rodríguez, C., Quiles, O. \& Herrera, L. (2005). Teoría y Práctica del Análisis de Datos cualitativos. Proceso General y Criterios de Calidad. Revista Internacional de Ciencias Sociales y Humanas, 15 (2), 133-154.

Sánchez Vidal, A. (1996). Psicología comunitaria, bases conceptuales y métodos de intervención. Barcelona, España: EUB.

Sarason, S. (1974). The psychological sense of community. Proaspects Community Psychology. San Francisco, CA: Jossey Bass.

Seminario, M. A. (2014). Sentido de comunidad, participación comunitaria y valores en lideres/as comu-
Diferencias intergeneracionales en el Sentido de Comunidad entre un grupo de niños y adultos mayores de la parcela de Cujacal en la ciudad de San Juan de Pasto - Colombia

nitarios/as en contextos de vulnerabilidad social (tesis para optar el título de Licenciado en Psicología con mención en Psicología Social). Pontificia Universidad Católica del Perú. Recuperado de: http://tesis.pucp.edu.pe/repositorio/bitstream/handle/123456789/5819/SEMINARIO_OBANDO_MIGUEL_ SENTIDO_LIDERES.pdf?sequence=1

Soto-Johnson, H., Yestness, N. \& Dalton, C. (2008): Students' perceptións of sense of community in abstract algebra: constributing factors and benefits. Eurasia journal of mathemactics, science $y$ tecnólogy education. 4 (4): 373 - 380. DOI: https://doi. org/10.12973/ejmste/75363

Spradley, J. P. (1980). Observación Participante. Madrid, España: Santillana.

Távara, M. (2012). Sentido de comunidad en un contexto de violencia comunitaria (tesis de maestría). Pontificia Universidad Católica del Perú. Recuperado de: http://tesis.pucp.edu.pe/repositorio/bitstream/handle/123456789/1651/TAVARA_VASQUEZ_MARIA_SENTIDO_VIOLENCIA.pdf?sequence=1

Vivaldi, F. \& Barra, E. (2012). Bienestar Psicológico, Apoyo Social Percibido y Percepción de Salud en Adultos Mayores. Terapia psicológica, 30(2), 23-29. https://dx. doi.org/10.4067/S0718-48082012000200002
2018, Vol. 12(23) 154-168 @The Author(s) 2018 Reprints and permission: www.americana.edu.co 